\title{
ON-OFF INTERMITTENCY IN RANDOMLY DRIVEN PARALLEL PUMPING
}

\author{
A. Krawiecki \\ Institute of Physics, Warsaw University of Technology \\ Koszykowa 75, 00-662 Warsaw, Poland
}

\begin{abstract}
A model of parallel pumping with many interacting parametric spin-wave pairs is investigated numerically. On-off intermittency is obtained in the time series of absorption when the rf field amplitude is varied randomly in time, slowly in comparison with the rf field frequency, and when its mean value exceeds slightly the parallel pumping instability threshold. If the interactions between parametrically and thermally excited spin waves are neglected, only one parametric spin-wave pair is strongly excited and exhibits intermittent behaviour. In the opposite case a packet of parametric spin waves with frequencies close to half the pumping frequency may be excited. This modifies quantitatively, but not qualitatively the intermittency characteristics in the presence of thermal noise.
\end{abstract}

PACS numbers: 05.45.+b, 76.50.+g, 75.30.Ds

On-off intermittency (OOI) is characterized by a time sequence of laminar phases, when the observed signal is close to zero, and chaotic bursts $[1,2]$. In nonlinear ferromagnetic resonance it can be observed in chaotic time series. of absorption if the rf field amplitude - constant in time - exceeds some critical value $[3,4]$. OOI may also occur if the rf field amplitude is varied randomly in time, slowly in comparison with the rf field frequency [5]. In this paper the latter possibility is discussed for the case of parallel pumping.

If the amplitude $h$ of the rf field $h \cos \omega t$ with frequency $\omega$, applied parallel to the dc field, is greater than the parallel pumping instability threshold $h_{\mathrm{thr}}$, pairs of parametric spin waves (SW) with frequency close to $\omega / 2$ and opposite wave vectors are excited [6]. This is also true if the amplitude $h(t)$ is a slowly varying, random or chaotic function of time, but in this case its average must exceed the instability threshold. In the following it is assumed that SW with wave vectors $k_{j}$ close in $k$-space and small detunings from half the pumping frequency $\Delta \omega_{k_{j}}=\omega_{k_{j}}-\omega / 2=j \Delta \omega, j=-N,-N+1, \ldots, N$, where $2 N+1$ is the number of SW pairs considered and $\Delta \omega$ is a parameter, may be excited. Equations of motion for the slowly varying in time parts $u_{k_{j}}$ of the complex SW pair amplitudes in the $S$-theory approximation [7] are as follows: 


$$
\dot{u}_{k_{j}}=\left(-\eta+\mathrm{i} \Delta \omega_{k_{j}}\right) u_{k_{j}}+\mathrm{i} \varepsilon(\mathrm{t}) \eta u_{k_{j}}^{*}+\mathrm{i}\left(2 u_{k_{j}} \sum_{i}\left|u_{k_{i}}\right|^{2}+\frac{S}{T} u_{k_{j}}^{*} \sum_{i} u_{k_{i}}^{2}\right)
$$

$$
+\xi_{\text {therm }} \text {. }
$$

Here, $\eta$ denotes phenomenological SW damping, $T, S$ are coefficients of diagonal four-mode interactions among SW, $\varepsilon(t)=h(t) / h_{\mathrm{thr}}$ is the time-dependent if field amplitude normalized to the parallel pumping instability threshold and $\xi_{\text {therm }}$ represents thermal noise (without the rf field, all SW are thermally excited to the level $\left.u_{\text {therm }}=\xi_{\text {therm }} / \eta\right)$. All SW have similar wave vectors and frequencies, therefore their damping and interaction coefficients are supposed to be the same. It is convenient to divide both sides of Eq. (1) by $\eta$ and work with renormalized time $t^{\prime}=\eta t$. Absorption in the sample is proportional to the sum of absolute values of $S W$ amplitudes.

Contrary to previous paper, where a model with one SW pair was considered [5], here the results for a group of $2 N+1=25$ modes are presented. It is assumed that $\varepsilon(t)=\varepsilon x(t)$, where $x(t)$ is a random square wave, i.e. a function changing its value randomly in the interval $[0,1]$ every $\Delta t^{\prime}$ renormalized time units, and $\varepsilon$ is the control parameter. The results below were obtained for $\Delta t^{\prime}=2.0, S / T=1.0$, but they remain qualitatively similar for other parameter values. Both cases of a discrete SW spectrum $(\Delta \omega / \eta=1.0)$ and of SW densely distributed in $\omega$-space $(\Delta \omega / \eta=0.025)$ were considered. The system of Eqs. (1) was solved numerically with the use of a fourth-and-fifth order Runge-Kutta method with permanent step size and error control.
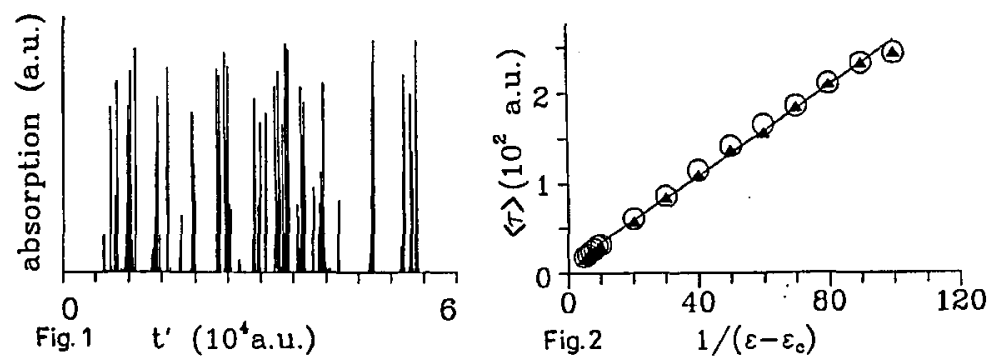

Fig. 1. Time series of absorption for $\varepsilon=2.033$.

Fig. 2. Scaling behaviour of the mean laminar phase length $\langle\tau\rangle$ with the control parameter $\varepsilon$ for $u_{\text {therm }}=0$ : triangles $-\Delta \omega=1.0$, circles $-\Delta \omega=0.025$.

If $\varepsilon>\varepsilon_{\mathrm{c}}=2.0$, i.e. when the mean value of the $\mathrm{r}$ f field amplitude exceeds the parallel pumping instability threshold, OOI in the time serics of absorption appears. Laminar phases can easily be distinguished from the bursts (Fig. 1). If $\xi_{\text {therm }}=0$ only one SW pair is excited during the bursts. For $\Delta \omega=1.0$ it is (for $\varepsilon<2.2$ ) the pair with $j=0$, whereas for $\Delta \omega=0.025$ the index $j$ of the excited pair increases from $j=0$ to $N$ with the rise of the rf field amplitude, hence for greater values of the control parameter amplitudes of SW pairs with greater detuning from half the pumping frequency exhibit bursts. Despite this difference, 
for a given value of the control parameter the mean laminar phase lengths in both cases are equal and decrease with the rise of the rf field amplitude proportionally to $\left|\varepsilon-\varepsilon_{\mathrm{c}}\right|^{-1}$ (Fig. 2); this is a scaling law characteristic of OOI [2]. It turns out that the probability distribution of the laminar phase lengths for given $\varepsilon$ follows a scaling law $P(\tau) \propto \tau^{-3 / 2}$, typical of OOI, too. These results are in quantitative agreement with the ones obtained within a model with one SW pair [5].
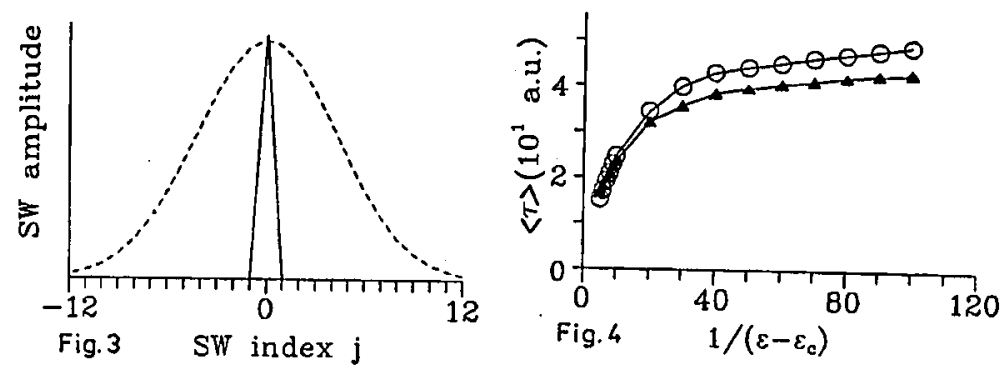

Fig. 3. Typical profiles of the spin-wave amplitudes during a burst for $u_{\text {therm }}=$ $1.0 \times 10^{-7}: \Delta \omega=1.0$ - solid line, $\Delta \omega=0.025$ - dashed line.

Fig. 4. As in Fig. 2, but for $u_{\text {therm }}=1.0 \times 10^{-7}$.

The situation changes for $\xi_{\text {therm }} \neq 0$. In the room temperature $u_{\text {therm }} \approx$ $10^{-8} \mathrm{~s}^{-1 / 2}$ in the units of Eq. (1) [5], but higher thermal excitation levels are also considered because then the intermittency characteristics differ sharply from the ones without thermal noise. If $\Delta \omega=1.0$ still one SW pair $j=0$ is strongly excited during the bursts, and other SW are only excited to the thermal level (Fig. 3). Ilowever, if $\Delta \omega=0.025$ a whole packet of $\mathrm{SW}$ with frequencies close to $\omega / 2$ is excited (Fig. 3). For $\varepsilon \approx 2.0$ the scaling law $\langle\tau\rangle \propto\left|\varepsilon-\varepsilon_{\mathrm{c}}\right|^{-1}$ is in both cases invalid, but the mean laminar phase length for $\Delta \omega=0.025$ is substantially shorter than for $\Delta \omega=1.0$ and the relative difference increases with increasing thermal noise (Fig. 4). The power law $P(\tau) \propto \tau^{-3 / 2}$ for the probability distribution of laminar phase lengths is replaced by an exponential fall-off for large $\tau$ (Fig. 5). The characteristic laminar phase length $\tau^{*}$ which separates these two scaling areas (signed by an arrow in Fig. 5) is again shorter for the system with $\Delta \omega=0.025$ than 1.0. In both cases $\tau^{*}$ scales with the noise intensity in a way which is characteristic of OOI $\tau^{*}=A \ln ^{2} u_{\text {therm }}+B \ln u_{\text {therm }}+C$, where $A, B, C$ are fitting parameters [8], different for different $\Delta \omega$ (Fig. 6). Thus the results for small $\Delta \omega$ differ from the ones obtained with the use of a model with one SW pair [5].

The excitation of a whole SW packet for small $\Delta \omega$ might have been expected on the basis of the investigation of the transient behaviour of the SW amplitudes after turning on the rf field [9]. It may be treated as an example of the widening of the phase space accessible to the system with OOI due to the presence of thermal noise. Many strongly excited SW pairs act as an additional source of noise in the system, which leads to the decrease in the mean laminar phase length and the exponential fall-off of the probability distribution of laminar phase lengths for shorter $\tau$. In systems with high-dimensional phase space this effect may cause 

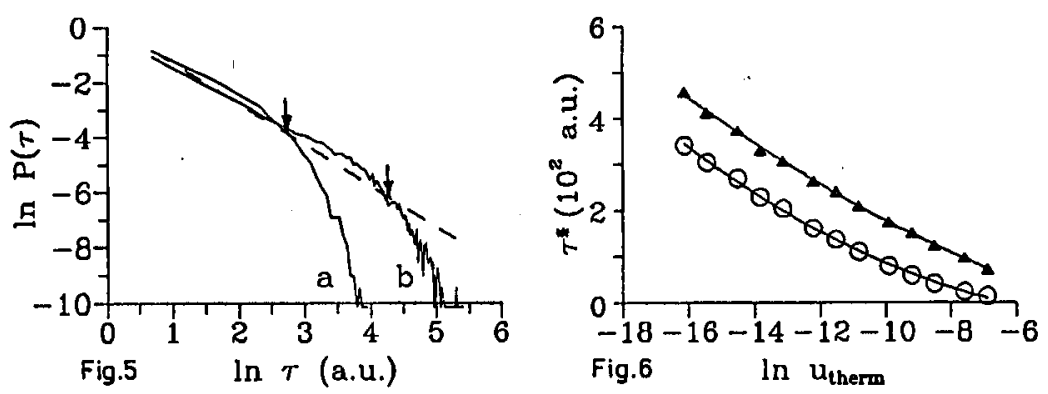

Fig. 5. Probability distribution of the laminar phase lengths for $u_{\text {therm }}=1.0 \times 10^{-3}$. Curve (a) is for $\Delta \omega=0.025$, curve (b) for $\Delta \omega=1.0$. Dashed line - least squares fit for $P(\tau) \propto \tau^{-3 / 2}$ for $u_{\text {therm }}=0$.

Fig. 6. Scaling behaviour of the characteristic laminar phase length $\tau^{*}$ (defined by arrows in Fig. 5) with the thermal noise $u_{\text {therm }}$ : triangles $-\Delta \omega=1.0$, circles $-\Delta \omega=$ 0.025 .

some difficulties in observing the characteristics of $\mathrm{OOI}$ in a wide range of the control parameter. Ilowever, it is interesting to note that in spite of dynamical and strongly nonlinear character of this additional noise the differences between the systems with different $\Delta \omega$ are of quantitative, not qualitative nature. This is an argument for the universal character of the scaling laws for OOI obtained in Refs. $[2,8]$ from the analysis of randomly driven logistic maps.

The author would like to thank Prof. Andrzej Sukiennicki and Prof. Jan J. Żebrowski for help and valuable discussions.

\section{References}

[1] N. Platt, E.A. Spiegel, C. Tresser, Phys. Rev. Lett. 70, 279 (1993).

[2] J.F. Heagy, N. Platt, S.M. Hammel, Phys. Rev. E 49, 1140 (1994).

[3] A. Krawiecki, A. Sukiennicki, Acta Phys. Pol. A 88, 269 (1995).

[4] F. Rödelsperger, A. Cenys, H. Benner, Phys. Rev. Lell. 75, 2594 (1995).

[5] A. Krawiecki, A. Sukiennicki, Acta Phys. Pol. A 89, 37 (1996).

[6] E. Schlömann, R.I. Joseph, J. Appl. Phys. 32, 1006 (1961).

[7] V. Zakharov, V. Lvov, S.S. Starobinets, Usp. Fiz. Nauk 114, 609 (1974) [Sov. Phys.-Usp. 17, 896 (1975)].

[8] N. Platt, S.M. Hammel, J.F. Heagy, Phys, Rev. Lett. 72, 3498 (1994).

[9] V.E. Zakharov, V.S. Lvov, S.L. Musher, Fiz. Tverd. Tela 14, 832 (1972) [Sov. Phys.-Solid State 14, 710 (1972)]. 